\title{
PSICOLOGOS DEL TESTIMONIO: ¿UN PERITAJE DE LUJO?
}

José JOAQUIN MIRA

Psicólogo 



\section{INTRODUCCION}

Hace unos meses apareció en uno de los diarios madrileños el caso de Luis González Aureoles, quien había permanecido en prisión durante veinte meses acusado de un delito que no había cometido. El error se debió, en esta ocasión, a que el propietario de la joyería donde se cometió el delito identificó como autor del mismo a González Aureoles y a que posteriormente confirmó su declaración al reconocerlo como culpable entre los miembros de una rueda de presos. Por fin, las protestas del abogado defensor acerca de las deficientes condiciones en las que se desarrolló el reconocimiento en la rueda y las numerosas e insistentes demandas de inocencia del acusado llevaron, el 29 de septiembre de 1982, a un careo entre denunciante y denunciado que concluyó con la declaración del joyero de que González Aureoles no era el atracador sino un cliente que "hacía tres años había comprado algo" en su joyería y por eso "le sonaba la cara".

Dentro de las investigaciones psicológicas sobre el testimonio de testigos se conoce este tipo de fenómeno como transferencia inconsciente (Glanville Williams, 1963, citado en Loftus, 1976, p. 93) y ha sido reproducido repetidas veces en estudios de laboratorio y de campo (Loftus, 1976, Deffenbacher, Brown y Sturgill, 1978, Hall, 1976b). En los trabajos realizados ha podido comprobarse, también, que si en la identificación inicial de un sospechoso, se identifica como autor del delito a un inocente, en las subsiguientes identificaciones el testigo tenderá a señalar de nuevo a ese sospechoso incorrecto (Gorenstein y Ellsworth, 1980) y que las personas son, normalmente, más capaces de reconocer una cara que de recordar donde la vieron (Brown, Deffenbacher y Sturgill, 1977).

¿Cómo puede llegar a ocurrir esto?, ¿cómo una persona a la que hemos visto en una situación muy concreta puede ser confundida con otra que vimos en otro lugar y en diferente situación?, ¿ pueden existir tipos tan similares entre sí o es más bien nuestra memoria la que nos hace verlos semejantes?, ¿es la psicología del testimonio capaz de responder a estos interrogantes? Sin duda conocer cómo funciona la memoria humana puede ayudarnos a explicar estas situaciones y a saber cómo un testigo recupera información de su memoria para declarar ante la policía o ante el juez, puesto que el funcionamiento de su memoria en estas situaciones no es diferente de cuando intenta recordar una película, la cara del camarero que le acaba de servir o a un amigo del colegio (Diges y Mira, 1983). 
Generalmente empleamos el término «memoria» para referirnos a nuestra capacidad, buena o pobre, de retener en la mente tanto las experiencias recientes como aquellas que constituyen nuestro pasado (Cofer, 1979).

El estudio experimental de la memoria se inicia con la publicación en 1885 de la obra de Hermann Ebbinghaus "Sobre la memoria» (Uber das Gedächtnis) donde se propuso determinar los mecanismos mentales básicos que intervienen en la memoria mediante los métodos de la ciencia natural. Esta orientación -heredada de los modelos asociacionistasconforma lo que se ha denominado "teoria de la huella", en la que se afirma que la memoria humana retiene huellas (engramas) de la experiencia original, que quedarian representadas en la memoria de cada individuo como unidades separadas (eslabones de una cadena) que carecerían en principio de toda relación pero que más tarde, como consecuencia de experiencias repetidas, se relacionarían de manera tal que la reactivación de un eslabón conduciría al enlace con otros eslabones de la cadena. Esta orientación, con el tiempo, ha dado lugar al desarrollo de modelos de aprendizaje verbal $y$, no precisamente, a estudios sobre la memoria tal y como antes fue definida.

En 1932 con los estudios de Sir Frederic Barttlet sobre la retención en circunstancias cotidianas, encontramos un enfoque distinto para investigar la memoria. Barttlet, consciente de las limitaciones del modelo empleado por Ebbinghaus, consideró que el recuerdo no es una función independiente y completamente distinta de la atención, la percepción, la imaginación o el pensamiento, muy al contrario constituye un todo en el cual resulta difícil determinar dónde empieza y termina cada proceso cognoscitivo. En su investigación hizo leer una leyenda esquimal a un grupo de individuos a quienes tras diversos intervalos de tiempo (desde pocos dias a muchos meses, incluso años) les pidió que contaran lo que recordaban de la historia. En esencia, encontró que los recuerdos estaban distorsionados, los propios individuos habían elaborado y racionalizado partes de la leyenda vulgarizando el texto para que fuera compatible con sus ideas $y$ experiencias previas. Para Barttlet la memoria es un proceso «esquemático", la gente recuerda aspectos generales y los detalles que se les presentan como más sobresalientes de los sucesos en los que intervienen, y a partir de éstos reconstruyen una versión que creen aproximada a la original. De esta forma, frente al modelo de reproducción de experiencias de Ebbinghaus, Barttlet propone un modelo de reconstrucción de experien- 
cias dando de esta forma cabida a una teoria de interpretación global del conocimiento humano (Mira y Diges, 1983a).

Esta segunda orientación se desarrolla definitivamente a partir de la década de los 50 , cuando empiezan a aparecer investigaciones sobre la memoria en las que se demuestra que el recuerdo del material original está mediado por las tendencias de respuesta del individuo y por sus experiencias previas, lo que da origen a que sucedan los errores de omisión o. comisión típicos del quehacer humano.

Desde esta perspectiva la memoria va a ser estudiada dentro de un proceso continuo en el que también tienen cabida la atención, la percepción y en esencia todos los procesos cognoscitivos. Entendiéndose, así, que el proceso de elaboración de información en el sujeto humano estaría dividido en tres fases interdependientes entre sí: adquisición, retención y recuperación de información, donde atención, percepción y memoria serían los tres momentos de un proceso continuo. Ante cualquier situación los individuos deben primero atender a la estimulación novedosa de su entorno y es entonces cuando deciden y seleccionan en qué estímulos y por cuanto tiempo se van a fijar (Broadbent, 1971), cuál va a ser la estrategia perceptual a emplear en ese caso y qué parte del entorno no recibirá nuestro interés. Atención y percepción deben ser entendidos como dos procesos limitados - sólo podemos atender a un número determinado de estímulos al mismo tiempo- y selectivos - nos fijamos siempre en lo más sobresaliente- que obligan a las personas a reconstruir los eventos en base al material retenido que, al sufrir este "filtraje previo», da lugar a que se cometan errores. Si además pensamos en una agresión o un delito donde las víctimas (testigos) se sienten amenazadas y bajo un fuerte estrés, es fácil entender que perderán parte de sus habilidades como observadores $y$, preocupándose más por su seguridad, no se fijarán en ciertos detalles del incidente que más tarde podrian ayudar a la policía en su trabajo y quizás los vacíos -errores de omisión - los "rellene" con ideas estereotipadas, hipótesis personales de cómo debió ocurrir, etc...-errores de comisión- (Levine y Tapp, 1973, Buckhout, 1974, Siegel y Loftus, 1978).

En términos generales se va a considerar que toda la información que llega a un observador, se centraliza en una «memoria de trabajo" que actúa como un proceso de control y almacén temporal que organiza y codifica esta nueva información en función de categorias permanentes de la memoria formadas en experiencias anteriores. De esta forma, la información es reciclada y puede sei alterada al menos de cuatro formas: 
1) la información original ambigua es codificada de forma consistente con los supuestos de la categoría empleada para interpretarla,

2) la información original no relevante o imposible de interpretar, en función de esa categoría, es eliminada (olvidada),

3) las características que no están presentes en la información original, pero que pertenecen a la categoría que se emplea para interpretarla, son añadidas a la información,

4) la información original se organiza de forma consistente con la categoría empleada para interpretarla, aunque difiera de su organización original. (Wyer y Srull, 1980).

Estas alteraciones en la información original se producen antes de su almacenamiento definitivo en la memoria, de tal modo que, al recuperar esta información, lo más probable es que el sujeto no pueda distinguir entre lo que es información original o lo que es su reconstrucción de los hechos (Bull y Clifford, 1979). De este modo, cabría redefinir lo que se va a entender por "memoria» de la siguiente manera: un proceso mediánte el cual se transforma, reduce, elabora, recupera y utiliza la información del mundo que el sujeto obtiene en su interacción con él (Neisser, 1967).

\section{¿POR QUE UNA PSICOLOGIA DEL TESTIMONIO?}

A nadie escapa que el testigo de algún suceso constituye una de las principales pruebas judiciales para decidir sobre la inocencia o culpabilidad de un sospechoso. Es por tanto la memoria - tal y como antes quedó definida - de los testigos el criterio decisivo para determinar que una persona quede libre o por el contrario, en prisión. Pero... ¿ es tan fiable la memoria de un testigo?

Las investigaciones pioneras en este campo surgen a finales del siglo pasado y van unidas estrechamente a los estudios sobre la memoria (Parker, 1980). En un principio surgen distintos autores que preocupados por la exactitud-inexactitud al contar relatos o sucesos buscan delimitar la seguridad que podemos tener en nuestra memoria. Se estudiará el efecto del intervalo de tiempo y la sugestión sobre el recuerdo, las diferencias en cuanto mediante recuerdo libre (narración) o por un método de interrogación (respuestas a preguntas sobre el hecho), o cómo puede influir el entrevistador en el relato final (Diges y Mira, 1983), temas que tienen mucho que ver con el elevado número de errores judiciales que informes de la época recogen (Borchard, 1932, citado en Hilgar y Loftus, 1979, p. 343).

Sin embargo, estas investigaciones son pronto abandonadas y olvida- 
das debido al empuje e implantación en toda la psicología del estudio del aprendizaje verbal y no será hasta la década de los 70 cuando a raíz de la irrupción en el panorama psicólogo del interés por el estudio de la memoria en sus ambientes naturales (Neisser, 1976), empezarán de nuevo a aparecer autores interesados en el testimonio y en delimitar las actuaciones de los testigos en base a criterios objetivos. Este nuevo interés ha calado hondo, especialmente en EE.UU. y Reino Unido, como lo demuestra una rápida ojeada a la revista Law and Human Behavior o a las publicaciones de la 41st división de la American Psychological Association (Psychology and Law) y la división de Criminología y Psicología Legal de la British Psychological Society. El desarrollo de esta área es ya una parte importante de todos los manuales sobre la memoria (Gruneberg y Morris, 1979, Neisser, 1982) y tema de interés en cursos y congresos (Gruneberg, Morris y Sykes, 1978), o incluso el tema monográfico a desarrollar como la Conferencia Internacional sobre Comportamiento de testigos que en junio de 1980 se desarrolló en Edmonton (Canadá) y donde se dieron cita los investigadores más prestigiosos en esta área de la psicología.

En la actualidad, la psicología del testimonio no se limita tan sólo al problema de la exactitud o fiabilidad del recuerdo, sino que entiende también que el testimonio hace referencia a un evento social y que se da en un contexto social que no sólo afecta a la cantidad de información retenida sino que ejerce su influencia en la calidad del recuerdo, esto es, la credibilidad del testigo (Mira y Diges, 1983a). Esta nueva orientación social, acorde con los planteamientos de Barttlet que antes expusimos, permite la interpretación de la actuación de los testigos en términos de un modelo del funcionamiento cognitivo humano (Nisbett y Ross, 1980, Mira y Diges, 1983a) que podría ser el siguiente: la información percibida acerca de cualquier suceso se categorizará en los guiones - secuencias de movimiento- y la información relativa a las personas actuantes en los personae -en donde se incluirían estereotipos y teorías implícitas de personalidad- y donde la información se alteraría según vimos antes. Cuando el sujeto-testigo se vea en la necesidad de recuperar esa información, recurirá para ello a los mecanismos de razonamiento (heurísticos) que hayan mostrado su eficacia en la elaboración de información precedente. Como Nisbett y Ross apuntan, éstos podrían ser: representatividad, que predice la pertenencia de una persona u objeto a una categoría dada, disponibilidad, que sirve de base para estimar la probabilidad de ocurrencia del hecho o su verosimilitud, vivacidad, definida por su interés emocional, concreción y capacidad imaginativa, y por la que la información estará más disponible en función de su proximidad sensorial, espacial y temporal. Es 
decir, si asumimos plenamente que la memoria no es una mera reproducción de eventos sino la reconstrucción en la mente del individuo de lo acaecido, nos vemos tentados a reinterpretar el aforismo de: «toda la verdad, sólo la verdad y nada más que la verdad..." por este otro: «...todo lo disponible, sólo lo representativo y nada más que lo muy vivido...".

\section{PROCEDIMIENTOS DE IDENTIFICACION DE PERSONAS}

El estudio de la memoria por los psicólogos permite diferenciar los procedimientos de identificación de personas en dos tipos:

a) aquellos procedimientos en los que al testigo se le pide que cuente todo lo que ha podido retener de cuanto ha oido y visto, es decir, todo lo que recuerda del suceso y las personas, $y$

b) aquellos procedimientos en los que al sujeto-testigo se le pide que reconozca al culpable de entre una serie de sospechosos.

En el primer caso estamos hablando de una tarea de recuerdo, que implica la búsqueda y recuperación de la información que se tiene almacenada, y en el segundo caso de reconocimiento, que se refiere a situaciones donde el sujeto debe decidir si un item determinado se le ha presentado antes o no. Ambos procedimientos implican unas habilidades y estrategias diferentes por parte de los sujetos que, además, suelen obtener mejores resultados cuando se trata de reconocimiento en vez de recuerdo (Garzón y Seoane, 1982).

\section{Recuerdo de personas}

Todos los procedimientos en uso de identificación de personas que requieren de los sujetos que recuerden cómo eran los individuos a identificar tiene en común que la cara es descompuesta en sus diferentes elementos (ojos, barbilla, labios, pelo, etc...) que luego, al ir combinándose adecuadamente, irán pareciéndose al original. Ejemplos de tales procedimientos son: los retratos-robots, las fotografías-robots, los sintetizadores de imágenes - normalmente por ordenador-, las caricaturas y los sistemas de rompecabezas (Davies, 1981).

De los sistemas de identificación de personas mencionados el más popular es, sin duda, la visión de un retrato-robot en el tablón de una comisaría. Su empleo data de 1959 y básicamente consiste en una serie de láminas transparentes en las que se encuentran dibujadas una gran variedad de rasgos faciales (en total 381 entre tipos de peinado, ojos, barbillas, 
labios, narices, etc..., además de que existen una serie de accesorios disponibles para completar los retratos, tales como gafas, sombreros, cicatrices, etc...) que al superponerse adecuadamente componen un retrato que se supone parecido al original. Su uso se encuentra muy difundido, sobre todo en EE.UU. país en el que se comercializa ', aunque como ha ocurrido en el Reino Unido este sistema está siendo reemplazado por el empleo de fotografías-robots (photofit), que si bien es esencia el mismo procedimiento, resulta un sistema bastante mejorado, lo que permite conseguir un mayor parecido con el original. La invención de este sistema se debe a Jacques Penry (1971) y está compuesto por 562 fotografías de rasgos faciales (como en el caso anterior), que están impresas en láminas transparentes que al ir combinándose en un marco-soporte especial componen una cara semejante a la original. Este sistema se diferencia del anterior en que la tarea de selección de las características faciales que más corresponden al modelo, por parte de los testigios, se encuentra facilitada por $\mu n$ índice visual en el que se encuentran representadas en miniatura las 562 características faciales según la categoría a la que pertenezcan (ojos, labios, etc...). Su comercialización se lleva a cabo tanto en EE.UU. como en el Reino Unido ${ }^{2}$ por lo que su uso está hoy totalmente estandarizado, y es precisamente por esta razón por la que se ha desarrollado en el mercado una segunda versión del retrato-robot (Identikit II) que en vez de dibujos emplea fotografías y en el que existen una mayor variedad de rasgos faciales para elegir.

En ambos procedimientos la tarea de los testigos es explicar al especialista de la policía cómo recuerdan que eran los rasgos faciales del sospechoso e indicar cuál de las láminas (en el caso del retrato-robot) o qué fotografía del índice visual (en el caso de la fotografía-robot) es la más parecida al original. El problema que se ha presentado en tales procedimientos es que parten de la hipótesis de que los testigos son capaces de componer una cara partiendo de sus elementos, hipótesis que no parece del todo correcta ya que existen pruebas de que las caras de las personas son percibidas y memorizadas como un todo y no mediante una aproximación de característica en característica (Davies, 1978, Christie y Ellis, 1981), tal como se desprende de las diferencias en la identificación de personas al presentárseles rasgos faciales aislados (solamente los ojos, la boca) o una combinación de ambos (los ojos y la boca, la frente y la nariz, etc...) que demuestran que aquí el todo es más que la suma de las partes (Fisher y Cox, 1975, Clifford y Bull, 1978).

La eficacia de estos sistemas de recuerdo de personas dependen de diversas circunstancias - como son los efectos de la interferencia en el 
recuerdo, el intervalo de tiempo transcurrido, el modo de representación, el proceso analítico ya sea como un todo o como una conjunción de elementos, los rasgos de presentación de las características faciales (Davies, 1981, Winograd, 1978, Ellis, Davies y Shepherd, 1977) - y, aunque son escasos los estudios de campo sobre su utilidad (los únicos estudios llevados a cabo se deben al grupo de Aberdeen -Davies, 1982-) parece sin embargo que su uso está recomendado (Maldin y Laughery, 1981, Laughery y Fowler, 1980).

Otros sistemas similares son los dibujos, caricaturas de los sospechosos, realizadas por dibujantes muy capacitados por lo que su uso está muy restringido y el empleo de sistemas de rompecabezas (strip systems) que consisten en series de láminas horizontales en las que la cara está diseccionada según diversos criterios, así por ejemplo en Francia se utilizan tres divisiones que corresponden a: pelo, ojos/nariz, boca/barbilla, en los EE.UU. en cambio existen normalmente cuatro divisiones: pelo, ojos, nariz, boca/barbilla y en Alemania Federal e Italia suelen considerarse cinco, pero en todos los casos, los componentes son similares: fotografías de caras reales.

Estos procedimientos, sin embargo, están siendo sustituidos aunque de una forma experimental por otros sistemas mucho más sofisticados, en los que se combinan sistemas ópticos altamente especializados que combinan diversas figuras (rasgos faciales) que un ordenador compone según las instrucciones del operador y los testigos. Ejemplos de tales sistemas son:Watsisface system, Sketch system, Minolta Montage Synthesizer (empleado por la policia japonesa con éxito) (Laughery, Rodes y Batten, 1981).

\section{RECONOCIMIENTO DE PERSONAS}

En cuanto a los procedimientos en los que se intenta reconocer a otras personas el más clásico y conocido es la rueda de presos o la búsqueda de un sospechoso entre fotografías de delincuentes habituales, procedimientos ambos que implican mecanismos psicológicos similares. Su estudio ha sido sistemáticamente realizado en otro lugar (Mira y Diges, 1983b), aunque conviene recordar que dichos procedimientos son excesivamente susceptibles a los efectos de la sugestión como en el caso de la transferencia inconsciente. De hecho, los aciertos obtenidos experimentalmente en tareas de reconocimiento de personas (Goldstein, 1977) resultan excesivamente inflados con los obtenidos en otras situaciones reales (Mira, 1983, Patterson, 1978, Buckhout, 1974). Resultado de todas estas investigaciones son las recomendaciones que, dirigidas a la policia y los tribunales, se han 
ido adoptando por los organismos competentes de varios países (Deffenbacher y Horney, 1981).

Por otro lado, estas diferencias han sido consideradas por algunos autores como prueba de que un entrenamiento adecuado puede ayudar a los sujetos a reconocer con más certeza a otras personas (Malpass, 1981). Los resultados en este sentido están, aún, en fase exploratoria, aunque parecen existir indicios de la eficacia de reinstaurar, previamente a la tarea de reconocimiento, las condiciones existentes cuando el testigo vio al sujeto en cuestión (Malpass y Devine, 1981).

\section{VARIABLES QUE AFECTAN LA IDENTIFICACION DE UNA PERSONA}

Son muchas las variables que pueden llegar a afectar a los sujetostestigos a la hora de identificar a alguien, tanto en la vida normal como cuando se trata de episodios delictivos. Algunas de estas variables ya fueron señaladas antes o estudiadas en otro lugar (Mira, 1983, Ellis, Davies y Shepherd, 1977) pero, sin duda, la clasificación más completa de las mismas se debe a Gary Wells (1978) para quien las variables que afectan a la identificación de una persona por un testigo se dividen en:

1) variables propias del sistema que se está empleando para identificar a los sospechosos. Son aquellas variables que la policía o los tribunales pueden manipular para optimizar la identificación (instrucciones, intervalo de tiempo, modo. de presentación, composición, etc...), y

2) variables a estimar, de las cuales únicamente podemos hipotetizar su efecto pero sin que podamos actuar sobre ellas (al menos directamente), afectando la ejecución de los testigos (tipo de crimen, diferencias individuales, actividad a la que han estado dedicados los testigos desde que sucedió el suceso, etc...).

Variables propias del sistema

G. Wells considera como variables del sistema aquellas que afectan al modo en que se organiza, desarrolla y ejecuta cada uno de los procedimientos de identificación. Son variables que se está en condiciones de manipular, y de hecho se hace, como lo demuestra que los retratos-robots sean sustituidos por las fotos-robots - que parecen más eficaces-y que, actualmente, se intente mejorar este procedimientos por medio de ordenadores. Esto es, el decidir utilizar un procedimiento u otro ya determina la calidad de la información que se obtendrá de los testigos. 
En el caso de los sistemas de recuerdo de caras se ha estudiado el efecto que sobre la composición final tienen las habilidades del operador (qué plazo de tiempo y número de inteṇtos son necesarios para que un operador de la policía adquiera soltura en determinado sistema) y por dónde resulta más eficaz comenzar la composición, si por el pelo, por la barbilla, o por el contorno de la cara, etc..., resultando más indicado comenzar por el pelo y los ojos y continuar por la nariz, la boca y la barbilla (Davies, 1982). Recordemos, en este sentido, las sugerencias de los estudios sobre la percepción que indicaban que las caras se recuerdan como un todo y que resulta mucho más difícil recordar aisladamente un rasgo facial. Así, otro problema adicional en estas técnicas es, precisamente, la amplitud de la muestra inicial de características faciales a partir de las cuales se va a componer la imagen del sujeto, siendo ésta la razón principal para abogar por el uso de ordenadores, que pueden presentar una visión global del rostro del sospechoso en la pantalla y que permiten al operador ir modificando ligera o radicalmente cualquier rasgo facial, manteniendo además en la memoria del ordenador las imágenes anteriores y toda la serie de modificaciones que han sido realizadas.

De igual forma, cuando se trata de reconocer a la persona vista entre otras (el caso de una rueda de presos) los investigadores se han interesado por determinar el número adecuado de componentes de la rueda (5, 6 u 8) (Malpass y Devine, 1983) o cuál debe ser el nivel de semejanza entre ellos para así determinar la imparcialidad - que ningún testigo esté más predispuesto a señalar a un componente de la rueda que a otro, salvo en base a su propia evidencia- del procedimiento de identificación (Mira y Diges, 1983b).

Las instrucciones dadas a los testigos también afectan a la calidad del testimonio. La idea de hacer revivir todo lo sucedido antes de proceder a reconocer al culpable (Malpass y Devine, 1981), el que las instrucciones se presenten de una forma objetiva y no sesgada (observando que el sospechoso puede o no estar en la rueda, p.e.) y el criterio que en la misma se asuma (criterio flexible: señalar si alguien se parece, o un criterio estricto: sólo indicar si se está seguro que es el culpable) juegan un papel importante en las decisiones de los testigos (Davies, Shepherd y Ellis, 1979), así como, también, utilizar dos ruedas de presos paralelas como medida precautoria de imparcialidad (Clifford y Bull, 1978).

Otra variable que también afecta la calidad del testimonio de los testigos es la influencia que el lenguaje puede llegar a ejercer sobre la memoria. No es lo mismo interrogar al testigo con una pregunta concreta que permitirle que cuente a su modo lo ocurrido o cómc vio al sospechoso 
(Hilgard y Loftus, 1979, Marquis, Marshall y Oskamp, 1972). La forma gramatical con que se hacen las preguntas ejerce una poderosa influencia en el recuerdo, hasta el punto que pueden distorsionarlo completamente, de tal modo que lleguen a afirmar cosas que jamás ocurrieron (Loftus y Zanni, 1975) o exagerar y deformar la realidad de acuerdo con la información o el estilo que la pregunta está sugiriendo (Loftus y Palmer, 1974). Este problema es aún más delicado por cuanto que la naturaleza reconstructiva de la memoria permite a los testigos que son interrogados con estas preguntas sesgadas que codifiquen esta información no original en el recuerdo que tienen de lo sucedido, de forma tal, que en los siguientes interrogatorios no podrán diferenciar lo que es su recuerdo original del recuerdo mediatizado por otros interrogatorios, asumiendo que ambos son el mismo (Cliford y Bull, 1978). Si a esta naturaleza reconstructiva de la memoria añadimos que a veces la policía tiene razones para sospechar de algún delincuente o estar muy motivada a obtener una respuesta positiva del testigo, es factible que esto se le está comunicando al mismo, aunque no de forma directa, sino al dejar pasar por alto la descripción de un rasgo, alguna foto, preguntando en ocasiones ¿está usted seguro?, con insinuaciones, mostrando interés por alguna respuesta, etc... (Buckhout, 1974, Smith, Pleban y Shaffer, 1982).

\section{Variables a estimar}

Al contrario de lo que sucedía con las anteriores, las variables a estimar son aquéllas que escapan a nuestra manipulación, si bien se puede estimar su magnitud a fin de conocer cómo pueden afectar al testimonio en general y a la identificación en particular.

Para facilitar su comprensión diferenciaremos los factores de la situación de los factores de los testigos.

- Fac. situación:

a) Las condiciones ambientales, físicas, en las que se desarrolló el incidente (distancia, oscuridad, niebla, influyen en la percepción que se tiene de los objetos hasta el punto de que Kuehn (1974) demostró que las declaraciones más pobres se producen cuando va a anochecer).

b) El tipo de delito que se trate, un rapto, una violación, etc...

c) El tiempo que dispone el testigo para observar (hasta un cierto límite suele existir una relación directa entre tiempo y calidad del testimonio) (Ellis, Davies y Shpherd, 1977). 
d) La actividad a la que se hayan dedicado los testigos hasta el momento de su declaración (los comentarios que se hacen mientras se espera a la policía o una película vista con anterioridad influyen en el testimonio) (Alper, 1976).

- Fac. testigos:

Las principales variaciones se deben a las diferencias individuales entre los testigos. Las mujeres, por ejemplo, son quienes con más seguridad reconocen una cara y, además, las caras que más fácilmente se recuerdan son las femeninas (Yarmey, 1979).

En cuanto a la edad, se ha comprobado que a medida que se desarroIla el niño su capacidad para identificar a los otros aumenta hasta aproximadamente los diez años, edad a partir de la cual el desarrollo de esta aptitud se torna lenta estabilizándose a los 17 años. A partir de esta edad parece que ya no se detectan diferencias entre los sujetos hasta que éstos no alcanzan los 60 y esta aptitud comienza a desaparecer (Mira, 1983).

Otras dimensiones en que los sujetos difieren entre sí son, personalidad, inteligencia, nivel de aprobación, necesidad de afiliación, sobre las cuales existen razones suficientes para sospechar que afectan al testimonio, si bien su estudio está en fase experimental (Mira, 1983).

\section{CONCLUSIONES}

Los errores de los testigos no son, sin duda, una novedad para un profésional de la justicia, aunque al profano le resulten cómicas algunas de las anécdotas provocadas por un testigo "de confianza» que resultó al final equivocado. Unas veces por olvidar algún detalle, otras por no fijarse en otro y en ocasiones por imaginar más de la cuenta, lo cierto es que los errores que cometen tienen su origen en la naturaleza reconstructiva de la memoria, como lo demuestran los estudios llevados a cabo desde principios de siglo par psicólogos del testimonio.

Se ha estudiado cómo el testigo atiende y percibe los sucesos que ocurren a su alrededor, cómo codifica la información que extrae en su memoria y de qué mecanismos se vale para recuperar esa información almacenada, cómo identifica a otras personas, bajo qué condiciones sociales actúa y qué diferencias hay entre los testigos (Mira, 1983). Proponiéndose dos ejes para delimitar la actuación de los testigos: a) fiabilidad del testimonio y b) credibilidad del testigo y su testimonio (Diges y Mira, 1983). Entre ambos se da el testimonio y se desarrolla toda una "escena" 
social que conducirá a un veredicto. Los hechos no sólo se almacenan en la memoria sino que, además, se tienen que reproducir en otros contextos sociales, donde al testigo se le reclaman habilidades que puede no posea. El estudio de las aptitudes de los individuos, de cómo actúan y en qué medida se les puede ayudar en su tarea es el objeto de la psicología y, en este caso, de la psicología del testimonio.

REFERENCIAS:

ALPER, A. y cols. Eyewitness identification: Accuracy of individual vs. composite recollections of a crime. Bulletin of the Psychonomic Society 1976, vol. 8 (2), 147-149.

BARTLETT, F. Remembering: a Study in Experimental and Social Psychology. Cambridge University Press. 1932.

BROADBENT, D. Decisión and Stress. Acadamic Press, London, 1971.

BROWN, E., DEFFENBACHER, K. \& STURGILL, W. Memory for faces and the circunstances of encounter. Journal of Applied Psychology, 1977, vol. 62 (3) 311-318.

BUCKHOUT, R. Eyewitness Testimony. Scientific American, 1974, 231, 23-31.

BULL, R. y CLIFFORD, B. Eyewitness Memory. En: Gruneberg y Morris (eds.) Applied Problems in Memory. Academic Press, London 1979.

CLIFFORD, B. y BULL, R. The Psychology of Person Identification. Routledge and Kegan Paul LTD. London 1978.

COFER, Ch. Estructura de la memoria humana. Ediciones Omega, BarceIona 1979.

CHRISTIE, D. y ELLIS, H. Photofit construction versus verbal descriptions of faces. Journal of Applied Psychology, 1981, vol. 66 (3), 358-363.

DAVIES, G. Face recognition: issues and theories. En: Gruneberg, Morris y Sykes (Eds.) Practical Aspects of Memory. Academic Press, London 1978.

DAVIES, G. Face Recall Systems. En: Davies, Ellis y Shepherd (Eds.) Perceiving and rememberin faces. Academic Press, London 1981.

DAVIES, G. Composite systems for recalling faces - helping the police with their enquiries-?. En: Trankell (Ed.) Reconstructing the past: the role of psychologists in criminal trials. Norstedts, Stockholm 1982.

DAVIES, G., SHEPHERD, J. y ELLIS, H. Similarity effects in face recognition. American Journal of Psychology, 1979 vol. 92, N. 3. 
DEFFENBACHER, K., BROWN, E. Y STURGILL, W. Some predictors of eyewitness memory accuracy. En: Gruneberg, Morris y Sykes (Eds.) Practical Aspects of Memory. Academic Press, London, 1978.

DEFFENBACHER, K. y HORNEY, J. Psycho-legal aspects of faces identification. En: Davies, Ellis y Sheperd (Eds.) Perceiving and Remembering Faces. Academic Press, London 1981.

DIGES, M. y MIRA, J. Psicólogos, Psicología y Testimonio. Análisis y Modificación de Conducta, 1983, vol. 9, n. ${ }^{\circ} 21,243-270$.

EBBINAHAUS, H. Memory. Teacher. College, New York, 1915. (Originalmente publicado en 1885).

ELLIS, H., DAVIES, G. y SHEPHERD, J. Experimental studies of face identification. National Journal of Criminal Defense, 1977, 3, 219-234.

FISHER, G. y COX, R. Recognizing human faces. Applied Ergonomics, 1975, 6, 104-109.

GARZON, A. y SEOANE, J. La memoria humana desde el procesamiento de la información. En: Delcaux y Seoane (Eds.) Psicologia Cognitiva y Procesamiento de la información. Pirámide, Madrid, 1982.

GOLDSTEIN, A. Fallibility of the eyewitness - Psychological Evidence-. En: Bruce Salas (Ed.) Psychology in the Legal Process. Spectrum Publications Inc., Flushing, NY 11366, 1977.

GORENSTEIN, G. y ELLSWORTH, P. Effects of choosing and incorrect photograph on a later identification by an eyewitness. Journal of Applied Psychology, 1980, vol. 65 (5), 616-622.

GRUNEBERG, M. y MORRIS, P. Applied Problems in Memory, Academic Press London, 1979.

GRUNEBERG, M., MORRIS, P. y SYKES, R. Practical Aspects of Memory. Academic Press, London, 1978.

HALL, D. Obtaining eyewitness identifications in criminal investigations: applications of social and experimental psychology. Dissertation Abstracts International, 1976, vol. 37 (5-B) 2569-2570.

HILGARD, E. y LOFTUS, E. Effective interrogation of the eyewitness. International Journal of Clinical \& Experimental Hypnosis, 1979, vol. 27 (4), 342-357.

KUEHN, L. Looking down a gun barrel: person perception and violent crime. Perceptual \& Motor Skill, 1974, 45, 157-164.

LAUGHERY, K. y FOWLER, R. Sketch artist and Identi-kit procedures for recalling faces. Journal of Applied Psychology, 1980, 65, 307-316. 
LAUGHERY, K., RHODES, B. y BATTEN, G. Computer-guided recognition and retrieval of facial images. En: Davies, Ellis y Shepherd (Eds.) Perceiving and remembering faces. Academic Press, London, 1981.

LEVINE, F. y TAPP, J. Psychology of criminal identification - the gap from Wade to Kirby-. University of Pennsylvania Law Review, 1973, V121 N5, 1079-1131.

LOFTUS, E. Unconscious transference in eyewitness identificaction. Law \& Psychology Review, 1976, vol. 2, 93-98.

LOFTUS, E. y PALMER, J. Reconstruction of automobile destruction: an example of the interaction between language and memory. Journal of Verbal Learning \& Verbal Behaviour, 1974, vol. 13, 585-589.

LOFTU'S, E. y ZANNI, G. Eyewitness testimony: the influence of the wording of a question. Bulletin of the Psychonomic Society, 1975, vol. 5 (1), 86-88.

MALPASS, R. Effective size and defendant bias in eyewitness identification lineups. Law and Human Behavior, 1981, vol. 5, n. ${ }^{\circ} 4,299-309$.

MALPASS, R. y DEVINE, P. Guided memory in eyewitness identification. Journal of Applied Psychology, 1981(a) vol. 66, n. ${ }^{\circ} 3$.

MALPASS, R. y DEVINE, P. Measuring the fairness foof eyewitness identification lineups. En: Lloyd-Bostock y Clifford (Eds.) Evaluating Witness Evidence. John Wiley \& Sons Ltd, London, 1983.

MARQUIS, K., MARSHALL, J. y OSKAMP, S. Testimony validity as a function of question form, atmosphere, and item difficulty. Journal of Applied Social Psychology, 1972, 2, 167-186.

MAULDIN, M. y LAUGHERY, K. Composite production effects on subsequent facial recognition. Journal of Applied Psychology, 1981, 66 (3).

MIRA, J. J. Memoria en la vida diaria: Un estudio experimental sobre testimonio. (Tesis de licenciatura) Pentalfa Ediciones (microficha), 1983.

MIRA, J. J. y DIGES, M. Psicología del testimonio: Un problema metodológico. Revista de Psicología General y Aplicada (en prensa).

MIRA, J. J. y DIGES, M. Tamaño, sesgos e imparcialidad de las ruedas de presos. Comunicación presentada en el / Congreso de Psicología de la excepcionalidad. Barcelona 1983b.

NEISSER, U. Cognitive Psychology, Appleton Century Crofts, N.Y. 1967.

NEISSER, U. Cognition and reality. Freeman, San Francisco 1976.

NEISSER, U. Memory observed. Remembering in natural contexts. Freeman \& Co. San Francisco 1982. 
NISBETT, R. y ROSS, L. Human Inference: Strategies and shortcomings of social judgement. Pren ice Hall, 1980.

PARKER, L. Legal Psychology - Eyewitness Testimony - Jury Behavior. Charles C. Thomas, Springfield, 1980.

PATTERSON, K. Person recognition: more than a pretty face. En: Gruneberg, Morris y Sykes (Eds.) Practical Aspects of Memory. Academic Press, London, 1978.

PENRY, J. Looking at faces and remembering them: a guide to facial identification. Elek books, London, 1971.

SIEGEL, J. y LOFTUS, E. Impact of anxiety and life stress upon eyewitness testimony. Bulletin of the Psychonomic Society. 1978, vol. 12 (6).

SMITH, J., PLEBAN, R. y SHAFFER, D. Effects of interrogator bias and a police trait questionnaire on the accuracy of eyewitness identification. Journal of Social Psychology. 1982, vol. 116, 19-27.

WELSS, G. Applied eyewitness-testimony research: system variable and estimator variable. Journal of Personality and Social Psychology, 1978, 36.

WINOGRAD, E. Encoding operations which facilitate memory for faces across the life span. En: Gruneberg, Morris y Sykes (Eds.) Practical Aspects of Memory. Academic Press, London, 1978.

WYER, R. y SRULL, T. The processing of social stimulus information: $A$ conceptual integration. En: Ostrom, Ebbesen, Wyer, Hamilton y Carlston (Eds.) Person Memory: The congnitive basis of social perception. Lawrence Erlbaum Associates, 1980.

YARMEY, A. The Psychology of Eyewitness Testimony. Free Press; New York, 1979.

NOT A S :

1 Identi-kit Company, 17985 Sky Park Circle, Suite C, California 92714, U.S.A.

2 Messr John Waddington of Kirkstall Ltd., Commercial Road, Leeds, LS5 3Aj, U.K. Sirchie Fingerprint Laboratories INC., P.O. Box 269, Moorestown NJ 08057 U.S.A.

\section{RESUMEN}

Dentro de las investigaciones sobre psicología del testimonio destaca, por el volumen de trabajos, el área de la identificación de personas. En este artículo se pretende dar una visión global de los diferentes procedi- 
mientos de identificación, aportando datos para juzgar su utilidad cuando son empleados por testigos para reconocer o recordar a una persona. Se sugiere que los errores de los testigos pueden ser interpretados al entender el funcionamiento de la memoria más como reconstrucción que como reproducción de sucesos, sugiriéndose como más conveniente para la interpretación de los datos actuales la teoría de conocimiento social de Nisbett y Ross (1980). Se aporta, también, una extensa bibliografía sobre este tema.

\section{SUMMARY}

According with the number of publications the most important area of eyewitness testimony is the research of person identification. In this paper we try to explain the methods for recognition and/or recall faces and the usefulness of this techniques when was used by eyewitness. Suggest that misidentification of eyewitness can be understanding if we see the human memory as event reconstruction more than event reproduction. We think that Nisbett \& Ross (1980) theorie about human inference as the most useful model to perform the investigations. Also is aviable an extensive bibliography about this area.

\section{RESUME}

D'Accord avec le número de plubications, l'ane plus important du témoignage sont les investigations sur l'identification des personnes. Dan cet article on explique le méthode pour reconnaître et/o nappeler visages, et l'utilité de cettes téchniques quand ils sont usé par témoins. On suggére que les erreurs des témoins ils penvent être entendus, si nous voyons la mémorie humaine plus reconstruction que comme reproduction dévérement. Nous pensons que la théorie de Nisbett y Ross (1980) c'est le modéle plus utile pour interpréter les investigations. On apporte une vaste documentation sur le theme. 